\title{
Efectos de la Fuerza lónica y el Tiempo de Remojo de Legumbres Secas sobre sus Propiedades Tecnofuncionales
}

\author{
Liliana Serna-Cock ${ }^{1}$, Omar V. Pabón-Rodríguez², Jesús D. Quintana-Moreno \\ (1) Universidad Nacional de Colombia Sede Palmira, Facultad de Ingeniería y Administración, \\ carrera 32 \# 12-00, Palmira, Valle, Colombia. (e-mail: Iserna@unal.edu.co; jdquintanam@unal.edu.co) \\ (2) Universidad Nacional de Colombia Sede Palmira, Facultad de Ciencias Agropecuarias, \\ carrera 32 \# 12-00, Palmira, Valle, Colombia (e-mail: ovpabonro@unal.edu.co)
}

Recibido Jul. 13, 2018; Aceptado Sep. 26, 2018; Versión final Nov. 20, 2018, Publicado Abr. 2019

\begin{abstract}
Resumen
En este estudio se determinó el efecto de la fuerza iónica y del tiempo de remojo de garbanzo Kabuli (Cicer arietinum L.), frijol cargamanto (Phaseolus vulgaris Cranberry Group), y arveja (Pisum sativum) sobre las cinéticas de absorción de agua y capacidad de retención de agua (CAA y CRA) de cada uno de los granos. Para esto se utilizaron soluciones de $0,0.1,2.6$, y $5.2 \mathrm{~mol}$ de iones de $\mathrm{NaCl} / \mathrm{L}$, en relación grano: solución (1:6). En los resultados se encontró que el tipo de grano, la fuerza iónica y el tiempo tuvieron efecto significativo sobre CAA y CRA. En garbanzo con la adición de $\mathrm{NaCl}$ a concentración de $0.1 \mathrm{M}$ se puede reducir el tiempo de remojo, de $8 \mathrm{~h}$ sin sal a $6 \mathrm{~h}$, obteniendo CAA y CRA de $0.8 \mathrm{~g}$ y $76.95 \%$ respectivamente. En relación con los granos frijol y arveja las mayores CAA Y CRA se obtuvieron con $14 \mathrm{~h}$ de remojo, sin la adición de $\mathrm{NaCl}$. La adición de $\mathrm{NaCl}$ en el remojo de granos puede disminuir el tiempo de remojo, pero la cantidad añadida influye sobre sus propiedades tecnofuncionales.
\end{abstract}

Palabras clave: propiedades de las proteínas; hidratación; fuerza iónica; granos secos; cinéticas de absorción

\section{Effects of the lonic Force and Time of Soaking of Dry Legumes on their Tecnofuncional Properties}

\begin{abstract}
In this study, the effects of ionic strength and soaking time of chickpea Kabuli (Cicer arietinum L.), bean cargamanto (Phaseolus vulgaris Cranberry Group), and pea (Pisum sativum) on the kinetics of water absorption and capacity of water retention (CAA and CRA) of each one of the grains were determined. To do this, solutions of $0,0.1,2.6$, and $5.2 \mathrm{~mol}$ of $\mathrm{NaCl} / \mathrm{L}$ ions were used, in relation grain: solution $(1: 6)$. In the results, it was found that grain type, ionic strength and time had a significant effect on CAA and CRA. In chickpeas with the addition of $\mathrm{NaCl}$ at a concentration of $0.1 \mathrm{M}$, the soaking time can be reduced from $8 \mathrm{~h}$ without salt to $6 \mathrm{~h}$, obtaining CAA and CRA of $0.8 \mathrm{~g}$ and $76.95 \%$ respectively. In relation to beans and peas, the largest CAA and CRA were obtained with $14 \mathrm{~h}$ of soaking, without the addition of $\mathrm{NaCl}$. The addition of $\mathrm{NaCl}$ in the soaking of grains can decrease the soaking time, but the added amount influences its technicalfunctional properties.
\end{abstract}

Keywords: properties of proteins; hydration; ionic strength; dry grains, absorption kinetics 


\section{INTRODUCCIÓN}

Las leguminosas secas están compuestas principalmente por carbohidratos y proteínas siendo los carbohidratos los compuestos mayoritarios de las leguminosas (64 al 68 \%) (Muhammad y otros. 2013). Los principales carbohidratos presentes en las leguminosas son el almidón y otros polisacáridos como celulosa, hemicelulosa, pectina, que constituyen la fibra dietética. Sin embargo, las leguminosas contienen cantidades pequeñas pero significativas de oligosacáridos como taninos, inhibidores de tripsina, inhibidores de proteasa, lectinas y ácido fítico, y, además, contienen carbohidratos simples como rafinosa, estaquiosa y verbascosa (Imran y otros, 2014). Estos carbohidratos simples presentes en las leguminosas secas son fermentados en el colon a ácidos grasos de cadena corta y $\mathrm{CO}_{2}$, ya que no pueden ser metabolizados en las primeras etapas de la digestión, por lo cual se consideran azúcares indeseables (Mederos, 2006). Rafinosa, estaquiosa y verbascosa son carbohidratos solubles en agua. El carácter de moléculas tóxicas solubles en agua hace que sea posible su eliminación a través del remojo de los granos. Por lo tanto, el adecuado remojo de estas leguminosas secas es indispensable para la eliminación de carbohidratos no asimilables, así como también es indispensable, la eliminación del agua del remojo antes del proceso de cocción (FAO, 2016). Sin embargo, la literatura científica es muy limitada para indicar los tiempos adecuados de remojo de leguminosas de alto consumo en poblaciones latinoamericanas. Y, a pesar de que científicamente se reconoce las bondades del remojo de los granos, esta práctica no es utilizada en forma masiva, dado que en las diferentes culturas latinoamericanas el conocimiento culinario se ha transferido de generación en generación y para algunas culturas esta práctica es inconveniente.

La capacidad de absorción (CAA) y retención de agua (CRA) son propiedades tecnofuncionales propias de las legumbres secas, que permiten determinar los tiempos adecuados de remojo de los granos, y están relacionadas directamente por el contenido proteico y la interacción proteína - agua (Sánchez, 2017). De igual manera, existe controversia si el agua de remojo para granos debería o no tener sal (Rodríguez y Fernández, 2015). El tema de la adición o no de sal en el agua de remojo de granos se ha abordado científicamente, sin embargo, los resultados siguen siendo controversiales. Por ejemplo, según Vanderleia y otros. (2014), la adición de sal en el agua de remojo disminuye el tiempo de cocción de los granos, arveja, frijol y garbanzo. En contraste, Chaparro y otros. (2011), indicaron que la adición de $\mathrm{NaCl}$ en el remojo de granos trae efectos negativos en la cocción. Según Rodríguez y Fernández (2003), es recomendable no adicionar sal antes del proceso de cocción dado que disminuye el tiempo de cocción y se requiere menor cantidad de sal para dar sabor. Además, la literatura indica que agregar sal antes del proceso de cocción reduce la calidad proteínica del grano (Chaparro y otros, 2011). La CAA y la CRA se ven afectadas entre otros factores, por la fuerza iónica (Traynham y otros., 2007). Las harinas obtenidas de leguminosas son fuente importante de proteínas, carbohidratos, minerales, vitaminas y fibra en la dieta, y sirven para mejorar las propiedades funcionales de otros productos (Aguilar y Vélez, 2013). La composición de proteínas y carbohidratos de los granos influencia las propiedades tecnofuncionales de las respectivas harinas y de los productos elaborados de ellas (Santiago y otros., 2018). Productos funcionales de harinas de leguminosas sirven para satisfacer las demandas de productos funcionales de la población vegetariana, y son utilizadas en las dietas mediterráneas dónde se estimula la ingesta de legumbres (Vaz Patto y otros., 2015). Para el desarrollo de estos productos y para el procesamiento industrial es indispensable conocer cuáles son los tiempos ideales de remojo en los cuales los granos alcanzan la mayor CAA y retención de agua CRA, y cómo influye la sal en el remojo de estos.

Por lo anterior, el objetivo de esta investigación fue determinar los tiempos de remojo de, garbanzo Kabuli (Cicer arietinum L.), frijol cargamanto (Phaseolus vulgaris Cranberry Group) y arveja (Pisum Sativum) utilizando diferentes concentraciones de fuerza iónica $(0,0.1,2.6$, y 5.2 moles de iones de $\mathrm{NaCl} / \mathrm{litro}$ de solución), a través de la medición de las cinéticas de CAA y la CRA. Además, determinar la fuerza impulsora de la movilidad del agua durante el remojo.

\section{MÉTODOS Y MATERIALES}

Los métodos y materiales son presentados en cinco subsecciones, método de selección de las leguminosas, proceso de remojo y preparación de soluciones, medición de las fuerzas impulsoras, cinéticas de absorción y retención de agua, y finalmente, se explica el diseño de experimentos utilizado en el estudio.

Selección de leguminosas: Se escogieron para posterior remojo, garbanzo (Cicer arietinum), frijol cargamanto (Phaseolus vulgaris Cranberry Group), y arveja verde (Pisum sativum) secos, empaquetados, de tamaño y peso promedio de 5,0654g arveja, 5,0532g frijol, 5,0612h garbanzo.

Remojo: Para el remojo se prepararon soluciones de $\mathrm{NaCl}$ de fuerzas iónicas de 0, 0.1, 2.6, y $5.2 \mathrm{~mol}$ de iones/l de solución, utilizando agua destilada a $\left.25^{\circ} \mathrm{C}, \mathrm{pH}: 7.0\right)$. Para el remojo se utilizó una relación 1:6 grano 
seco:agua. La solución con fuerza iónica cero se utilizó como control (agua sin adición de sal). El remojo se realizó a $25^{\circ} \mathrm{C}$ por 24 horas.

Medición de la fuerza impulsora de las soluciones: Para verificar la fuerza impulsora del agua se midió la actividad de agua $\left(\mathrm{a}_{\mathrm{w}}\right)$ (Aqualab water activity meter series 3 TE®, U.S.A) tanto a las soluciones salinas como a los granos secos

Cinéticas de capacidad de absorción de agua (CAA) y capacidad de retención de agua (CRA): La cinética de CAA se midió durante 24 horas siguiendo la metodología modificada de (McWatters y otros., 2002). Durante las primeras 8 horas se tomaron muestras cada hora, posteriormente, se tomaron muestras a las 14, 16, 20, y 24 horas. En los diferentes tiempos se retiraron por triplicado granos remojados de los diferentes tratamientos. Los granos se secaron suavemente con papel absorbente y se pesaron en balanza analítica (Mettler Toledo, USA). En cada tiempo se calculó la CAA, mediante la ecuación 1, y los resultados se expresaron como g de $\mathrm{H}_{2} \mathrm{O}$ absorbida/g de grano seco.

$\mathrm{CAA}=\frac{\text { peso grano remojado }(\mathrm{g}) \text {-peso grano seco }(\mathrm{g})}{\text { peso grano } \operatorname{seco}(\mathrm{g})}$

A partir de los datos de CAA se construyeron para cada grano curvas CAA versus tiempo. Cada punto en la curva correspondió al promedio de los triplicados.

Para medir la cinética de CRA a los granos a los cuales se les midió la CAA se sometieron a una fuerza de centrifugado a 3200 rpm por 30 min. En cada tiempo se calculó el porcentaje de la CRA mediante la ec. 2.

$\% \mathrm{CRA}=\frac{\text { peso de grano remojado centrifugado }(\mathrm{g})-\text { peso del grano seco }(\mathrm{g})}{\text { peso de grano seco }(\mathrm{g})} \times 100$

Diseño de experimentos: se utilizó un diseño factorial de 3*4 con medidas repetidas en el tiempo, así: Factor tipo de grano con tres niveles (garbanzo, frijol, y arveja). Factor fuerza iónica en las soluciones de remojo (0, $0.1,2.6,5.2$ moles de iones de $\mathrm{NaCl} / \mathrm{I}$ de solución). Las variables de respuesta fueron CAA, CRA. A partir de los gráficos CAA y CRA vs tiempo se calcularon los tiempos apropiados de remojo de cada grano. Las mediciones de CAA y CRA se realizaron en 12 tiempos. Se utilizó el software estadístico SAS 9.3 para analizar los resultados, y para identificar diferencias entre los tratamientos se realizó comparación entre medias con $\mathrm{p}<0.05$.

\section{RESULTADOS Y DISCUSIÓN}

Esta sección se divide en cuatro subsecciones que presentan y explican los resultados obtenidos en el estudio en CAA y CRA de las tres legumbres secas.

Cinéticas de CAA y CRA: En las figuras 1,2 y 3 se presentan las cinéticas de CAA de garbanzo, arveja y frijol a diferentes concentraciones de fuerza iónica. El ANOVA mostró que el tipo de grano, la fuerza iónica y el tiempo de remojo influyeron en la CAA. La fuerza iónica y la CAA presentaron relación inversa. Se presentaron diferencias significativas en la CRA en los diferentes tratamientos por efecto del tipo de grano, la adición de sal y el tiempo de remojo. Las Tablas 1, 2 y 3 presentan los promedios y las desviaciones estándar de la CRA de los diferentes granos en los diferentes tiempos de remojo.

Las cinéticas de CAA y CRA de los garbanzos en todas las concentraciones de fuerza iónica se caracterizaron por presentar una fase exponencial y una fase de meseta, no se presentó fase de latencia. En la fase exponencial los granos de garbanzo absorbieron agua a velocidad constante, en esta etapa la cantidad de agua absorbida aumentó al aumentar los tiempos de remojo. La fase de meseta en el remojo comenzó cuando presumiblemente el agua llenó todos los espacios capilares e intermoleculares libres (Shafaei y otros, 2016). Las cinéticas de CAA y CRA en frijol y arveja remojados sin sal y con la menor concentración de sal no mostraron fase de latencia, sin embargo, a concentraciones de 2,6 y 5,2 moles de iones de $\mathrm{NaCl} / \mathrm{l}$ de solución presentaron fase de latencia, fase logarítmica y fase estacionaria. Shafaei y otros. (2016) indicaron que la CAA de agua de legumbres como garbanzo Kabuli y otras variedades y frijol de tres variedades, depende del tiempo de remojo y de la temperatura del agua de remojo. Kinyanjui y otros. (2015), reportaron que en la cinética de absorción de agua del frijol entero remojado a $25^{\circ} \mathrm{C}$ sin adición de sal se presenta fase de latencia. La diferencia encontrada entre las cinéticas de este autor y la del presente estudio (sin adición de sal) se puede explicar porque en el estudio de Kinyanjui y otros. (2015), se tomaron muestras a los 30 minutos de remojo, mientras que en la presente investigación se tomaron muestras cada hora durante las primeras 8 horas de remojo, por lo cual en la gráfica cinética no se aprecia la fase de latencia. 
CAA y CRA de garbanzo: Sin adición de sal los garbanzos presentaron la mayor CAA a 14 horas de remojo (82\%), sin embargo, en estas condiciones no se obtuvo la mayor CRA. Los valores de CAA desde la hora 8 hasta la hora 24 no presentaron diferencias estadísticamente significativas, por lo cual se puede decir que para garbanzo son suficientes 8 horas de remojo sin adición de sal para obtener valores cercanos al $80 \%$ de CAA. Sin embargo, con adición de sal a concentración de 0.1 moles de iones de $\mathrm{NaCl} / /$ de solución la mayor CAA de garbanzo fue del $80 \%$ en tan solo 6 horas de remojo, obteniéndose para ese mismo tiempo la mayor CRA posible $(76.95 \%)$.

Es importante resaltar que a la hora 7 se perdió el $23 \%$ de esta CRA (59.35\%), lo que indica que, en el remojo con sal, el tiempo resulta ser altamente relevante. Las diferencias en la CAA encontradas en garbanzo con 14 horas de remojo sin sal y 6 horas de remojo con sal no fueron significantes, (ver Figura 1 y Tabla 1), por lo cual, se puede concluir que el remojo de garbanzos se hace más eficiente con la adición de sal a concentraciones de 0.1 moles de iones de $\mathrm{NaCl} / \mathrm{l}$ de solución, dado que disminuye el tiempo de remojo en dos horas, sin embargo, sobrepasar este tiempo resulta indeseable. Los tratamientos sin sal requieren 16 horas para alcanzar CRA de 74\%. Además, un comportamiento destacable ocurrió a las 24 horas de remojo, donde las cinéticas de CAA y CRA no presentaron diferencias significantes entre los tratamientos, lo que indica que si los garbanzos se remojan con sal o sin sal por $24 \mathrm{~h}$ se obtienen valores muy cercanos en la CAA y en CRA. Sería necesario evaluar qué ocurriría durante la cocción de estos granos remojados a las condiciones de mayor CAA y CRA, ya que esta investigación no tuvo ese alcance.

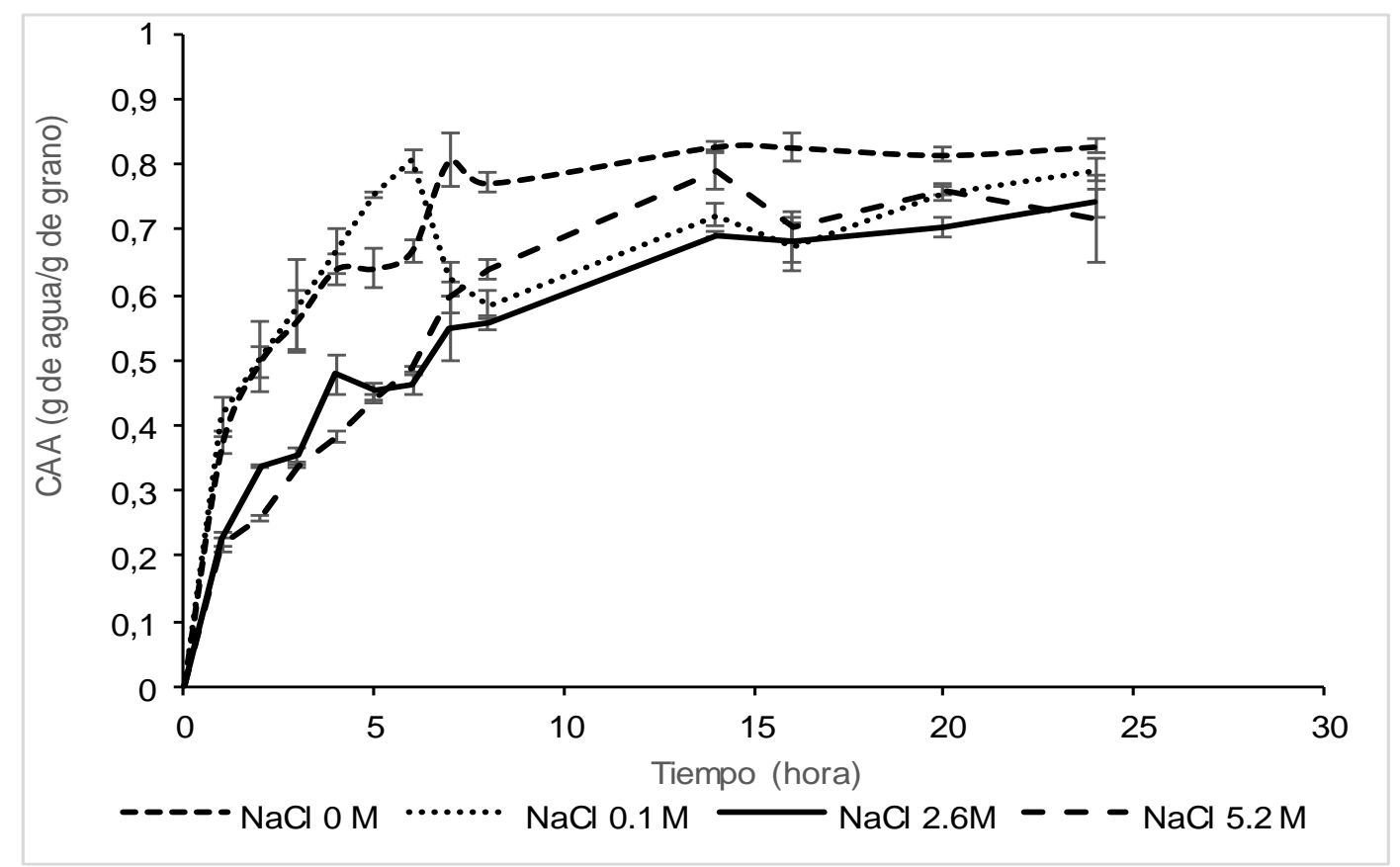

Fig. 1: Cinética del efecto de la fuerza iónica sobre la capacidad de absorción de agua (CAA) de garbanzo (Cicer arietinum)

Tabla 1: Capacidad de retención de agua (CRA) de garbanzo (promedio de tres replicas) a diferentes concentraciones de fuerza iónica.

\begin{tabular}{|c|c|c|c|c|}
\hline $\begin{array}{l}\text { Concentración Moles de } \\
\text { lones NaCl/L solución }\end{array}$ & Tiempo (h) & $\begin{array}{c}\text { \%CRA } \\
\text { Garbanzo }\end{array}$ & $\begin{array}{l}\text { Concentración Moles de } \\
\text { lones NaCl/L solución }\end{array}$ & $\% C R A$ Garbanzo \\
\hline \multirow{12}{*}{0} & 1 & $34,72 \pm 1,30$ & \multirow{12}{*}{0,1} & $41,52 \pm 2,59$ \\
\hline & 2 & $46,50 \pm 3,27$ & & $45,70 \pm 10,60$ \\
\hline & 3 & $51,74 \pm 3,21$ & & $56,93 \pm 6,95$ \\
\hline & 4 & $57,88 \pm 3,42$ & & $64,58 \pm 2,47$ \\
\hline & 5 & $59,44 \pm 2,51$ & & $72,53 \pm 0,73$ \\
\hline & 6 & $58,51 \pm 1,82$ & & $76,95 \pm 2,15$ \\
\hline & 7 & $71,46 \pm 4,67$ & & $59,35 \pm 1,86$ \\
\hline & 8 & $71,87 \pm 2,08$ & & $55,69 \pm 1,06$ \\
\hline & 14 & $71,18 \pm 0,47$ & & $64,54 \pm 0,66$ \\
\hline & 16 & $74,31 \pm 1,0$ & & $63,22 \pm 5,49$ \\
\hline & 20 & $72,01 \pm 1,44$ & & $63,53 \pm 1,60$ \\
\hline & 24 & $72,73 \pm 2,02$ & & $69,87 \pm 0,96$ \\
\hline
\end{tabular}


Tabla 1 (continuación)

\begin{tabular}{|c|c|c|c|c|}
\hline $\begin{array}{l}\text { Concentración Moles de } \\
\text { lones } \mathrm{NaCl} / \mathrm{L} \text { solución }\end{array}$ & Tiempo (h) & $\begin{array}{c}\% C R A \\
\text { Garbanzo }\end{array}$ & $\begin{array}{c}\text { Concentración Moles de } \\
\text { lones } \mathrm{NaCl} / \mathrm{L} \text { solución }\end{array}$ & $\% C R A$ Garbanzo \\
\hline \multirow{12}{*}{2,6} & 1 & $21,94 \pm 0,63$ & \multirow{12}{*}{5,2} & $20,24 \pm 1,57$ \\
\hline & 2 & $31,81 \pm 0,83$ & & $24,65 \pm 0,71$ \\
\hline & 3 & $34,17 \pm 1,21$ & & $33,00 \pm 1,166$ \\
\hline & 4 & $45,34 \pm 3,47$ & & $37,58 \pm 1,19$ \\
\hline & 5 & $44,68 \pm 1,48$ & & $43,58 \pm 1,92$ \\
\hline & 6 & $45,49 \pm 1,49$ & & $47,97 \pm 0,97$ \\
\hline & 7 & $51,16 \pm 3,37$ & & $58,34 \pm 2,19$ \\
\hline & 8 & $54,63 \pm 0,54$ & & $63,39 \pm 1,06$ \\
\hline & 14 & $66,33 \pm 0,45$ & & $77,15 \pm 2,31$ \\
\hline & 16 & $67,76 \pm 3,03$ & & $69,42 \pm 2,01$ \\
\hline & 20 & $69,19 \pm 1,53$ & & $72,90 \pm 1,55$ \\
\hline & 24 & $73,78 \pm 1,22$ & & $71,01 \pm 6,46$ \\
\hline
\end{tabular}

CAA y CRA de frijol: El frijol mostró la mayor CAA y la mayor CRA a $20 \mathrm{~h}$ de remojo sin sal, obteniéndose en este tiempo más de su peso en agua, y reteniendo casi la totalidad (96.7\%). No obstante, entre 14 y 24 horas de remojo los valores de CAA y CRA no fueron estadísticamente diferentes (Ver Figura 2 y Tabla 2).

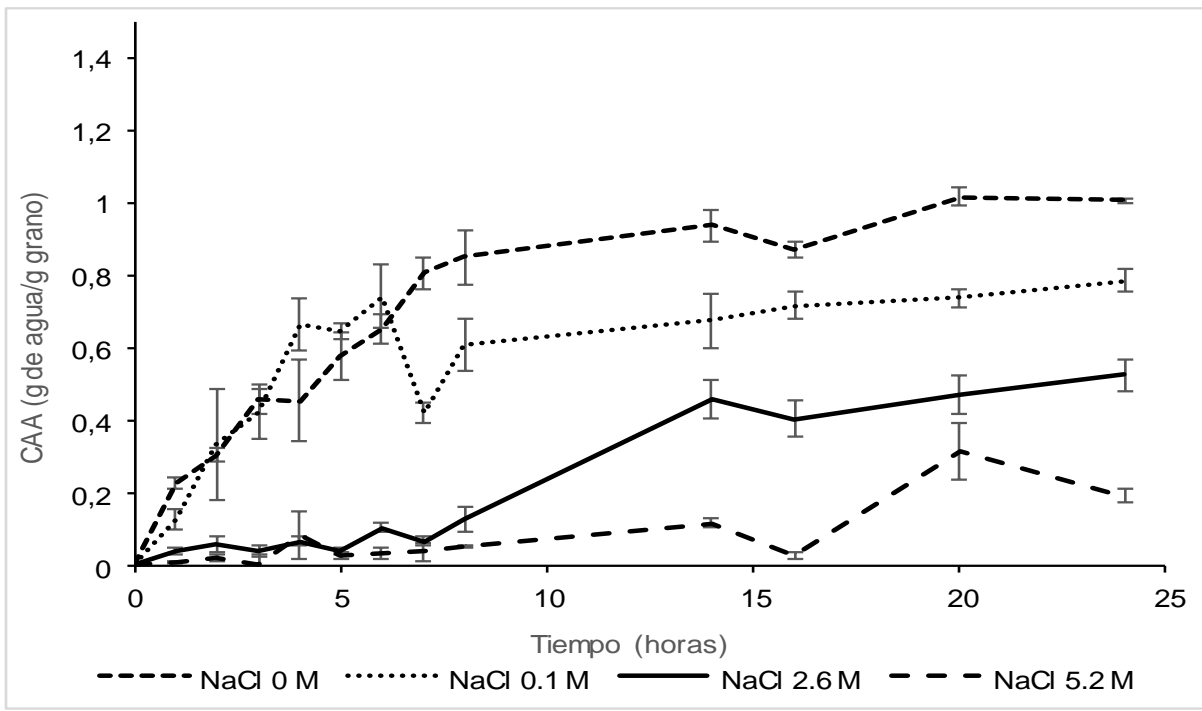

Fig. 2: Cinética del efecto de la fuerza iónica sobre la capacidad de absorción de agua (CAA) de frijol (Phaseolus vulgaris Cranberry Group)

Tabla 2: Capacidad de retención de agua (CRA) de frijol (promedio de tres replicas) a diferentes concentraciones

\begin{tabular}{|c|c|c|c|c|}
\hline $\begin{array}{l}\text { Concentración moles de iones } \\
\qquad \mathrm{NaCl} / \mathrm{L} \text { solución }\end{array}$ & Tiempo (h) & $\% C R A$ Frijol & $\begin{array}{l}\text { Concentración moles de iones } \\
\mathrm{NaCl} / \mathrm{L} \text { solución }\end{array}$ & $\% C R A$ Frijol \\
\hline \multirow{12}{*}{0} & 1 & $21,80 \pm 0,86$ & \multirow{12}{*}{0,1} & $14,38 \pm 3,41$ \\
\hline & 2 & $24,03 \pm 8,14$ & & $30,67 \pm 13,93$ \\
\hline & 3 & $46,47 \pm 4,71$ & & $43,56 \pm 6,52$ \\
\hline & 4 & $45,30 \pm 12,19$ & & $64,72 \pm 5,87$ \\
\hline & 5 & $54,69 \pm 5,13$ & & $63,02 \pm 2,74$ \\
\hline & 6 & $60,54 \pm 4,34$ & & $70,39 \pm 8,13$ \\
\hline & 7 & $75,97 \pm 3,68$ & & $40,55 \pm 2,77$ \\
\hline & 8 & $87,94 \pm 4,11$ & & $57,42 \pm 7,48$ \\
\hline & 14 & $89,70 \pm 3,80$ & & $66,09 \pm 6,86$ \\
\hline & 16 & $83,05 \pm 1,7$ & & $68,22 \pm 3,40$ \\
\hline & 20 & $96,77 \pm 1,14$ & & $70,06 \pm 1,34$ \\
\hline & 24 & $90,66 \pm 4,76$ & & $73,94 \pm 2,83$ \\
\hline \multirow{2}{*}{2,6} & 1 & $3,13 \pm 0,41$ & \multirow{2}{*}{5,2} & $0,63 \pm 0,17$ \\
\hline & 2 & $5,37 \pm 1,78$ & & $1,81 \pm 1,08$ \\
\hline
\end{tabular}


Tabla 2 (Continuación)

\begin{tabular}{|c|c|c|c|c|}
\hline $\begin{array}{c}\text { Concentración moles de iones } \\
\mathrm{NaCl} / \mathrm{L} \text { solución }\end{array}$ & Tiempo (h) & $\% C R A$ Frijol & $\begin{array}{l}\text { Concentración moles de iones } \\
\qquad \mathrm{NaCl} / \mathrm{L} \text { solución }\end{array}$ & $\% C R A$ Frijol \\
\hline \multirow{10}{*}{2,6} & 3 & $3,72 \pm 1,11$ & \multirow{10}{*}{5,2} & $0,34 \pm 2,77$ \\
\hline & 4 & $6,66 \pm 1,28$ & & $3,94 \pm 10,20$ \\
\hline & 5 & $4,22 \pm 0,86$ & & $2,37 \pm 0,55$ \\
\hline & 6 & $9,40 \pm 1,72$ & & $2,64 \pm 1,18$ \\
\hline & 7 & $6,61 \pm 1,43$ & & $2,72 \pm 2,22$ \\
\hline & 8 & $13,48 \pm 3,29$ & & $5,40 \pm 0,43$ \\
\hline & 14 & $45,32 \pm 4,23$ & & $10,38 \pm 1,20$ \\
\hline & 16 & $40,27 \pm 4,77$ & & $2,73 \pm 0,75$ \\
\hline & 20 & $46,01 \pm 4,35$ & & $29,58 \pm 5,20$ \\
\hline & 24 & $53,45 \pm 4,50$ & & $19,10 \pm 2,22$ \\
\hline
\end{tabular}

Con la adición de sal a concentración de 0.1 moles de iones de $\mathrm{NaCl} / /$ de solución la CAA descendió significativamente a 74\% en 6 horas de remojo. Sin embargo, favoreció la CRA, obteniéndose 10\% más de CRA, comparado con el mismo tiempo sin la adición de sal. Mayores concentraciones de sal no favorecieron ni CAA ni la CRA del frijol. Por lo anterior se puede afirmar que son suficientes 14 horas de remojo del frijol sin sal para obtener valores máximos de CAA y CRA.

CAA y CRA de arveja: Para arveja son necesarias 24 horas de remojo sin sal para alcanzar la mayor CAA (95\%) y la mayor CRA (93\%), sin embargo, al igual que el frijol, entre 14 y 24 horas de remojo los valores de CAA no fueron estadísticamente diferentes, por lo cual 14 horas son suficientes para el remojo de este grano. La adición de 0.1 moles de iones de $\mathrm{NaCl} / \mathrm{l}$ de solución favoreció la CAA y la CRA en las primeras 4 horas de remojo. Mayores concentraciones de sal no favorecieron ni la CAA ni la CRA de la arveja Con concentraciones de sal de 0.1 moles de iones de $\mathrm{NaCl} /$ de solución ocurrió lo mismo que en frijol, la CAA máxima alcanzada fue de tan solo $72 \%$ en 6 horas de remojo y de 69\% de CRA (Ver Figura 3 y Tabla 3). Al igual que frijol se puede indicar que sin la adición de sal son suficientes 14 horas de remojo para un eficiente remojo de arveja.

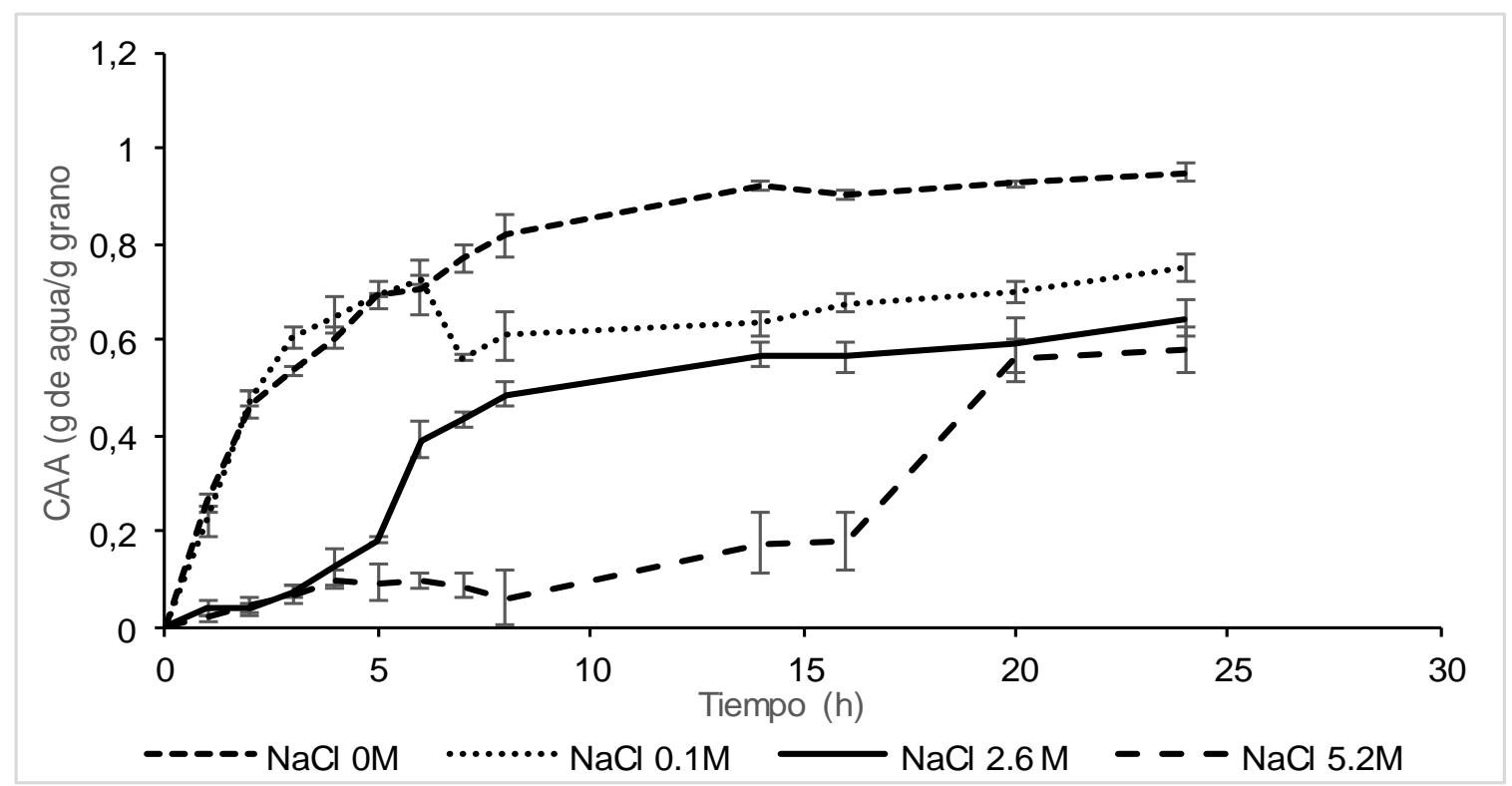

Fig. 3: Cinética del efecto de la fuerza iónica sobre la capacidad de absorción de agua (CAA) de arveja (Pisum sativum)

De otro lado, en los granos evaluados en las horas de remojo 4, 5, 6, la CAA fue mayor a concentraciones de sal de 0.1 moles de iones de $\mathrm{NaCl} / /$ de solución comparado con la CAA de los granos sin la adición de sal, siendo más marcada la diferencia en la hora 4 para frijol y arveja, tiempo en el cual ambos granos alcanzaron CAA de $66 \%$. Sin sal a 4 horas de remojo el frijol y la arveja solo alcanzaron CAA de $45 \%$. En garbanzo la mayor diferencia en los tratamientos con sal y sin sal se observó a la hora 6 , donde del grano alcanzó el $80 \%$ de CAA con la adición de la mínima cantidad de sal evaluada, en contraste, sin la adición de sal en $5 \mathrm{~h}$ de remojo, el garbanzo alcanzó sólo el $66 \%$ de CAA. 
Tabla 3: Capacidad de retención de agua (CRA) de arveja (promedio de tres replicas) a diferentes concentraciones de fuerza iónica

\begin{tabular}{|c|c|c|c|c|}
\hline $\begin{array}{l}\text { Concentración moles de iones } \\
\mathrm{NaCl} / \mathrm{L} \text { solución }\end{array}$ & Tiempo (h) & $\% C R A$ Arveja & $\begin{array}{l}\text { Concentración moles de iones } \\
\qquad \mathrm{NaCl} / \mathrm{L} \text { solución }\end{array}$ & $\% C R A$ Arveja \\
\hline \multirow{3}{*}{0} & 1 & $25,95 \pm 4,01$ & \multirow{3}{*}{0,1} & $20,15 \pm 3,07$ \\
\hline & 2 & $42,10 \pm 3,99$ & & $45,80 \pm 3,62$ \\
\hline & 3 & $53,28 \pm 1,05$ & & $57,61 \pm 2,78$ \\
\hline \multirow{9}{*}{0} & 4 & $60,15 \pm 2,58$ & \multirow{9}{*}{0,1} & $63,91 \pm 3,99$ \\
\hline & 5 & $69,12 \pm 0,81$ & & $66,41 \pm 3,50$ \\
\hline & 6 & $68,43 \pm 5,37$ & & $69,42 \pm 2,96$ \\
\hline & 7 & $76,04 \pm 2,29$ & & $54,36 \pm 1,95$ \\
\hline & 8 & $80,43 \pm 4,31$ & & $59,15 \pm 4,59$ \\
\hline & 14 & $90,44 \pm 0,87$ & & $61,60 \pm 2,06$ \\
\hline & 16 & $89,73 \pm 0,91$ & & $67,47 \pm 1,97$ \\
\hline & 20 & $90,49 \pm 1,39$ & & $69,37 \pm 1,94$ \\
\hline & 24 & $93,04 \pm 1,37$ & & $75,04 \pm 2,50$ \\
\hline \multirow{3}{*}{2,6} & 1 & $2,86 \pm 1,32$ & \multirow{3}{*}{5,2} & $1,20 \pm 0,34$ \\
\hline & 2 & $3,12 \pm 1,66$ & & $3,58 \pm 1,698$ \\
\hline & 3 & $6,92 \pm 0,64$ & & $5,68 \pm 1,79$ \\
\hline \multirow{9}{*}{2.6} & 4 & $12,45 \pm 3,422$ & \multirow{9}{*}{5,2} & $8,192 \pm 1,23$ \\
\hline & 5 & $16,89 \pm 0,77$ & & $8,58 \pm 1,78$ \\
\hline & 6 & $34,75 \pm 6,92$ & & $8,19 \pm 0,97$ \\
\hline & 7 & $42,87 \pm 1,73$ & & $8,119 \pm 2,66$ \\
\hline & 8 & $47,53 \pm 2,57$ & & $5,74 \pm 5,25$ \\
\hline & 14 & $55,87 \pm 2,45$ & & $17,07 \pm 6,27$ \\
\hline & 16 & $55,33 \pm 3,35$ & & $17,406 \pm 6,02$ \\
\hline & 20 & $56,76 \pm 5,54$ & & $54,50 \pm 5,04$ \\
\hline & 24 & $61,63 \pm 4,21$ & & $53,58 \pm 4,93$ \\
\hline
\end{tabular}

Chaparro y otros. (2011), midieron la CAA de semillas de vitabosa, una leguminosa de la familia del frijol y encontraron valores de $0.71 \pm 0.06 \mathrm{~g}$ de agua/g de semillas, estos valores están por debajo de los obtenidos en el presente estudio para frijol cargamanto. En arveja verde se reporta CAA de 0,24 g/semilla, en arveja de campo $0,15 \mathrm{~g} / \mathrm{semilla}$, y en arveja de mar 0,005 g/semilla (Chavan y otros., 2001). Estos valores no son comparables con nuestro estudio dado que no se especifica el peso de la semilla. Seena y otros. (2005), midió la CAA de semillas como Canavalia cathartica $(0,15 \mathrm{ml} / \mathrm{semilla})$ y Canavalia marítima $(0,05 \mathrm{ml} / \mathrm{semilla})$ pertenecientes a la familia de las leguminosas obteniendo 0.15 y $0.05 \mathrm{~g}$ de agua por semilla. Al igual que en arveja estos resultados no son comparables con los obtenidos en nuestra investigación dado que las unidades reportadas en el estudio de Seena y otros. (2005) corresponden a la CAA de cada semilla y no de gramo de semilla, lo que se considera un error experimental, dado que no reportan el peso de las semillas.

La CAA y CRA son consideradas propiedades funcionales o tecnofuncionales de las proteínas, las cuales pueden ser potenciadas por los carbohidratos. La CAA se refiere a la tendencia que tienen las moléculas de agua para asociarse con los compuestos hidrofilicos o algunos materiales celulares, esta depende del material no acuoso, la salinidad, el pH y la temperatura del alimento, por otra parte, la CRA es un término usado para referirse a la eficacia que tienen algunas macromoléculas (proteínas), para atrapar altas cantidades de agua, evitando la exudación de esta, y su liberación el alimento es sometido a una fuerza o corte, además, de que se comportara como el agua inicial del alimento, congelándose, eliminándose por secamiento, y conservando su capacidad disolvente, también, ayuda a medir el rendimiento de un alimento, entre mayor sea su retención de agua, mejor es su calidad (Fennema, 2010). La CAA y CRA requieren de la interacción proteína-agua, estas propiedades dependen del balance hidrófilo-hidrofóbico de los aminoácidos en las moléculas de proteína, de conformaciones estéricas, y del tamaño y tipo de proteína (Jitngarmkusol, y otros., 2008). Cuando los granos son remojados, las moléculas de agua entran en interacción con las proteínas propias de los granos, estas tienen grupos cargados que pueden formar interacciones ion-dipolo, las amidas y carboxilos formados en la conformación de enlaces peptídicos, además, algunos aminoácidos cuentan en su cadena lateral con grupos iónicos como las amidas, carboxilos e hidroxilos (ácido aspártico, ácido glutámico, lisina y arginina) con la característica de formar puentes de hidrogeno, en adición a esto, el agua puede unirse a residuos no polares dónde se induce una interacción dipolo-dipolo (Marc, 2016).

Las leguminosas tienen la capacidad de sintetizar y almacenar proteínas por ello son especies vegetales nutricionalmente importantes (Zia-Ul-Haq y otros., 2007), y esas proteínas pueden presentar propiedades tecnológicas útiles en la formulación de alimentos. Para las leguminosas en general se estima que el contenido en proteínas se encuentra entre el 20-40\% (Li Day, 2013). En garbanzo el contenido de proteínas oscila entre 17 al $22 \%$ bs, la mayoría de ellas globulinas que se consideran proteínas de reserva (70\%), el 
resto corresponde a albúminas y glutelinas (Aguilar y Vélez, 2013) además, los aminoácidos mayoritarios en este grano son Glu 18.7g, Asp 13.5g, Arg 10.4g, Leu 8g, Lys 7.2g, de 100g de AA (Cortés, 2016), los cuales 49.8g (Glu, Asp, Arg, y Lys) son hidrofilicos, ayudando en las propiedades tecnofuncionales de hidratación y retención, en frijol pinto son Glu 153, Asp 111, 89 Leu, 78 Lys, y 66 Arg de 1000 residuos de AA (San, 2013) y en arveja son Glu 33.3g, Asn 20.2g, Arg 15.7g, Lys 14.4g, 13,1 Leu, por Kg de grano (Koivunen, 2016), donde la Leucina es el único aminoácido hidrofóbico dentro de los cinco mayoritarios en los tres granos, el contenido de proteína en base seca oscila entre 21.3 a $27.6 \%$ bs para frijol rojo, y para arveja es de $28.6 \%$ bs (Muhammad y otros., 2013).

La adición de sales afecta las propiedades tecnofuncionales de los productos que contienen gran cantidad de proteína (Rodríguez, 2015). La adición de concentraciones de $\mathrm{NaCl}$ de $0.1 \mathrm{M}$ favoreció la CAA y la CRA en garbanzo, y esto es una evidencia de las diferencias entre los aminoácidos que conforman la proteína de los granos evaluados. En términos generales se conoce que a concentraciones bajas de sal en el medio acuoso, la proteína tiende a ligar mejor el agua, mejorando su CAA (Canchi y García, 2013). La adición de pequeñas cantidades de sal disminuyó el coeficiente de actividad de la proteína presente en el garbanzo (interacciones proteína-proteína) y su CAA aumentó, esto se explica por las fuerzas de atracción entre los iones de las proteínas y los iones del $\mathrm{NaCl}$. En garbanzo, frijol y arveja a mayores fuerzas iónicas 2.6 y 5.2 mol de iones /l de solución en el agua de remojo, el agua se ligó con los iones de la sal y fue por ello por lo que estos tratamientos tuvieron las menores CAA y CRA. Lo que ocurrió en estos casos se explica porque la fuerza de los enlaces entre agua y el $\mathrm{NaCl}$ son más fuertes que los enlaces de hidrógeno entre moléculas de agua (Rivera y otros., 2001).

La CRA fue substancialmente diversa entre los diferentes granos, fuerzas iónicas y tiempos de remojo. La CRA en granos remojados sin adición de sal a $24 \mathrm{~h}$ de remojo osciló entre 72 a $93 \%$. El hecho de que los granos no hubieran alcanzado un $100 \%$ de CRA indica que todos los granos durante el secado sufrieron daños en sus puntos de unión con el agua ya que el secado produce efecto sobre los puntos electrostáticos que interactúan con el agua. Un incremento en la temperatura de los granos ocasiona cambios físicos y químicos que pueden reducir el número de sitios activos donde se unen las moléculas de agua con otras moléculas (Teresa, 2016). El mismo fenómeno lo explica Mohamed y otros., (2004), de forma diferente, los autores indican que al aumentar la temperatura se incrementa el estado de excitación de las moléculas de agua, aumentando la distancia entre ellas, este hecho disminuye las fuerzas de atracción entre estas moléculas. Las leguminosas utilizadas en esta experimentación fueron granos secos en los cuales se desconoce los métodos de secado utilizados ya que fueron obtenidos del comercio local, sin embargo, en países latinoamericano las prácticas de secado de granos como fríjol y arveja se hacen en su mayoría mediante secadores solares (Mesa y otros., 2014), con lo cual se esperaría que tuvieran menor CRA comparado con los garbanzos, los cuales son secados en secadores convencionales dónde se hace más fácil el control de la temperatura, y por ende, se favorece la conservación de la calidad del grano. Las temperaturas utilizadas durante el secado de granos son capaces de romper puentes de hidrogeno (Vojdani, F. 2006) afectando la estabilidad de las interacciones no-covalentes de la estructura tridimensional de las proteínas, dado que se eleva la entalpía de la molécula y se rompe el delicado balance de los enlaces que mantienen el equilibrio (Feyzi y otros., 2017).

La CAA se explica también por los diferenciales en las actividades de agua (aw) de las soluciones de remojo y de los granos. Las aw de las soluciones de remojo de fuerzas iónicas de 0, 0.1, 2.6 y 5.1moles de iones/l de solución fueron de $1,0.955,0.925$ y 0.809 respectivamente, y las aw de los granos secos fueron de 0.554 , 0.615 , y 0.530 para garbanzo, frijol y arveja respectivamente la diferencia entre estos valores para las legumbres se explica porque se utilizaron granos comerciales con diferentes contenidos de humedad. El proceso de secado de los granos ayuda a romper los puentes de hidrogeno (Vojdani, F. 2006) que ligan agua. El valor de $a_{w}$, refleja la disponibilidad de agua que tiene un alimento para las reacciones enzimáticas, o el crecimiento de microorganismos, y depende de la composición porcentual de compuestos químicos hidrofilicos e hidrofóbicos del grano (Diana, 2014). El diferencial de aw ejerció una fuerza impulsora que hizo que el agua migrara desde las soluciones de remojo con aw más altas hacia los granos con aw más bajas, y esta es la causa de que los granos tras el remojo se hidraten y no se deshidraten, es decir, no pierdan el agua con la que han quedado después del secado.

\section{CONCLUSIONES}

En esta investigación se demostró que el tipo de grano, la fuerza iónica y el tiempo de remojo influyeron en la CAA y en la CRA. Se determinó que el remojo de garbanzos se hace más eficiente con la adición de sal a concentraciones de $0.1 \mathrm{M}$, dado que se disminuyó el tiempo de remojo en dos horas, pasando de 8 horas sin adición de sal a 6 horas con adición de sal, además, a estas condiciones de obtiene la máxima CRA. En el remojo con sal de garbanzo, frijol y arveja el tiempo fue altamente relevante. El frijol y la arveja requieren mínimo de 14 horas de remojo y la adición de sal solo tiene efectos positivos en las primeras horas de remojo. 


\section{AGRADECIMIENTOS}

A la Universidad Nacional de Colombia por la financiación de esta investigación y a Norwin Steven Rodríguez Hurtado y Cristian David Arcila Chacua por el apoyo en la experimentación.

\section{REFERENCIAS}

Aguilar, R. y J. Vélez, Propiedades Nutricionales y Funcionales del Garbanzo (Cicer arietinum L.), Temas selectos de Ingeniería de Alimentos, 7(2), 25-34 (2013)

Canchi, D. R. y A. E. García, Cosolvent Effects on Protein Stability, Annual Review of Physical Chemistry, 64, 273-293 (2013)

Chaparro, S.P., J. Gil e I.D. Aristizábal, Efecto de la Hidratación y la Cocción en las Propiedades Físicas y Funcionales de la Harina de Vitabosa (Mucuna deeringiana). VITAE, 18(2), 133-143, (2011)

Chavan, U., D. McKenzie y F. Shhidi, Functional Properties of Protein Isolates from Beach Pea (Lathyrus maritimus L.), Food Chem., 74 (2), 177-187 (2001)

Cortés, G.I., C. Megías y otros tres autores, Purification of free arginine from chickpea (Cicer arietinum) seeds, ISSN: 1609-9117, Food Chemistry, 192(1), 114-118 (2016)

Diana P.N., A.A. Alfredo y S.V. Héctor, Adsorción de Vapor de Agua de Bioplásticos Elaborado con Harina de dos Variedades de Yuca (Manihot esculenta Crantz), ISSN: 0718-0764, Rev. Información Tecnológica, 25(6), 23-32, (2014)

FAO. Organización de las Naciones Unidas para la Alimentación y la Agricultura, Beneficios de las Legumbres para la Salud (2016)

Fennema, O. R., S. Srinivasan y K. L. Parkin, Química de los Alimentos, 3르 Ed., 34-35, Acribia, España (2010)

Feyzi, S., M. Varidi, F. Zare y M.J Varidi, A Comparison of Chemical, Structural and Functional Properties of Fenugreek (Trigonella foenum graecum) Protein Isolates Produced Using Different Defatting Solvents, International Journal of Biological Macromolecules, 105(1), 27-35 (2017)

Imran, H., A. Asif y otros tres autores, Nutricional and Health Perspectives of Beans (Phaseolus Vulgaris L.): An Overview, ISSN: 1040-8398, Critical Reviews in Food Science and Nutrition, 54(5), 580-592 (2014)

Jitngarmkusol, S., J. Hongsuwankul y K. Tananuwong, Chemical Compositions, Functional Properties, and Microstructure of Defatted Macadamia Flours, Food Chemistry, 110(1), 23-30 (2008)

Kinyanjui, P., D. Njoroge y otros cuatro autores, Hydration Properties and Texture Fingerprints of Easy- and Hard-to-Cook Bean Varieties, Food Science \& Nutrition, 3(1), 39-47 (2015)

Koivunen, E., K. Partanen y otros cuatro autores, Digestibility and energy value of pea (Pisum sativum L.), faba bean (Vicia faba L.) and blue lupin (narrow-leaf) (Lupinus angustifolius) seeds in broilers, Animal Feed Science and Technology 218(1), 120-127 (2016)

Li Day, Proteins from land plants- Potential resources for human nutrition and food security, Trends in Food Science \& Technology, 32, 25-42 (2013)

Marc R.C., F. Elena, G.G Núria y C.B. Hanne, Multiple spectroscopic approach to elucidate water distribution and watereprotein interactions in dry-cured ham after high pressure processing, ISSN: 0260-8774, Rev. Journal of Food Engineering, 169, 291-297 (2016)

McWatters, K.H., M.S. Chinnan y otros cuatro autores, Functional, Nutritional, Mycological and Akara-Making Properties of Stored Cowpea Meal. J. Food Sci., 67 (6), 2229-2234 (2002)

Mederos, Y., Indicadores de la Calidad en el Grano de Frijol (Phaseolus vulgaris L.), Cultivos Tropicales, 27(3), 55-62 (2006)

Mesa, Y. M., G. Domínguez, Y. Rodríguez y J. León, Use of an Artisan Solar Grain Dryer to Dry Soybeans and Black Beans Grains, Revista Ciencias Técnicas Agropecuarias, 23(4), 17-20 (2014)

Mohamed, L. A., M. Kouhila y otros tres autores, Moisture Sorption Isotherms and Heat of Sorption of Bitter Orange Leaves (Citrus auratium), J. Food Eng., 67, 491-498 (2004)

Muhammad, A., W.R. Lloyd, A. Rashida y N. Mian, Application and Opportunities of Pulses in Food System: a Review, Critical Reviews in Food Science and Nutrition, 53 (11), 1168-1179 (2013)

Rivera, J.M., G.D. Blanco, A.C. Agrasal y L.R. Olmo, Bioquímica Estructural Conceptos y Test, Editorial Tébar, 2009 - 386 páginas (página de consulta 84), (2001)

Rodríguez, L. y X.E. Fernández, Los frijoles (Phaseolus vulgaris): Su Aporte a la Dieta del Costarricense. Acta Médica Costarricense, 45, 120-125 (2003)

Rodríguez, S. y X.E. Fernández, Prácticas de Preparación y Conservación de Frijoles en Familias Costarricenses, Agron. Mesoam. 26(1), 153-164 (2015)

Rodríguez, P., R. Jimy, M. Ticona y T. Jessica, Influencia de la adición de sal y tiempo-temperatura de cocción en la capacidad de retención de agua en carne de alpaca (Vicugna pacos) utilizando tecnología de cocción bajo vacío, Tesis de pregrado, Universidad Nacional de San Agustin, Perú (2015) 
Sánchez, M.N., J.R. Ruiz., G.O. Dávila y C.M. Jiménez, Propiedades Tecnofuncionales y Biológicas de Harina, Aislado y Fracciones Proteicas Mayoritarias de Semillas de Inga Paterno, Journal of food, 15(3), $400-408$ (2017)

Santiago, R.D., C.J. Figueroa, M.J. Véles y R. Salazar, Physicochemical Properties of Nixtamalized Black Bean (Phaseolus vulgaris L.) flours, Food Chemistry 240, 456-462 (2018)

Seena, S. y K. Sridhar, Physicochemical, Functional and Cooking Properties of Under Explored Legumes, Canavalia of the Southwest Coast of India, Food Res Int. Aug., 38 (7), 803-814 (2005)

Shafaei S.M., A.A. Masoumi y H. Roshan, Analysis of Water Absorption of Bean and Chickpea during Soaking Using Peleg Model, Journal of the Saudi Society of Agricultural Sciences, 15, 135-144 (2016)

Teresa, A.B., M.Z. Stella y otros cuatro autores, Influencia de las variables de secado en la calidad organoléptica de Bocaditos Salados a base de Amaranto, ISSN: 0718-8706, Av. Cien. Ing., 7(4), 47-56 (2016)

Traynham, T.L., J.M. Deland., A.L. Carriquiry y L.A. Johnson, Evaluation of Wáter-holding Capacity for Whea-Soy Flour Blends, Journal of the American Oil Chemists Society, 84, 151-155 (2007)

Vanderleia, S., R.C. Silvia, C. Divair y S. Silvio, Processing Parameter Optimization for Obtaining Dry Beans Witch Reduced Cooking Time, Food Science and Technology, 56 (1), 49-57 (2014)

Vaz Patto, M.C., R. Amarowicz y otros cincos autores, Achievements and Challenges in Improving the Nutritional Quality of Food Legumes, Critical Reviews in Plant Sciences, 34 (1-3), 105-143 (2015)

Vojdani, F., Solubility. In G. M. Hall, Methods of Testing Protein Functionality, $1^{\text {a }}$ Ed., Blackie Academic \& Professional, 11-60, Great Britain, St. Edmundsbury (2006)

Zia-UI-Haq, M., S. Iqbal y otros cuatro autores, Nutritional and Compositional Study of Desi Chickpea (Cicer arietinum L.) Cultivars Grown in Punjab, Pakistan. doi:10.1016/j.foodchem.2007.05.004, Food Chemistry, 105 (4), 1357-1363 (2007) 